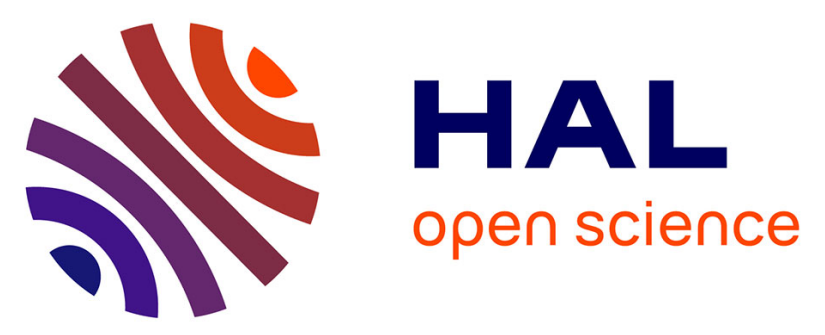

\title{
Integrated Silicon Nitride Horizontal Long Period Grating for Refractometric Gas Sensing applications
}

Clement Deleau, Han Cheng Seat, Helene Tap, Frederic Surre, Olivier D Bernal

\section{- To cite this version:}

Clement Deleau, Han Cheng Seat, Helene Tap, Frederic Surre, Olivier D Bernal. Integrated Silicon Nitride Horizontal Long Period Grating for Refractometric Gas Sensing applications. 2020 IEEE International Instrumentation and Measurement Technology Conference (I2MTC), May 2020, Dubrovnik, Croatia. pp.1-6, 10.1109/I2MTC43012.2020.9129170 . hal-03547247

\section{HAL Id: hal-03547247 \\ https://hal.science/hal-03547247}

Submitted on 31 Jan 2022

HAL is a multi-disciplinary open access archive for the deposit and dissemination of scientific research documents, whether they are published or not. The documents may come from teaching and research institutions in France or abroad, or from public or private research centers.
L'archive ouverte pluridisciplinaire HAL, est destinée au dépôt et à la diffusion de documents scientifiques de niveau recherche, publiés ou non, émanant des établissements d'enseignement et de recherche français ou étrangers, des laboratoires publics ou privés. 


\section{Integrated Silicon Nitride Horizontal Long Period Grating for Refractometric Gas Sensing applications}

\author{
Clément Deleau \\ LAAS-CNRS \\ University of Toulouse, INP \\ Toulouse, France \\ clement.deleau@laas.fr \\ Frédéric Surre \\ School of Mathematics, Computer \\ Science and engineering \\ University of London \\ London, United Kingdom \\ f.surre@ieee.org
}

\author{
Han-Cheng Seat \\ LAAS-CNRS \\ University of Toulouse, INP \\ Toulouse, France \\ seat@toulouse-inp.fr \\ Olivier Bernal \\ LAAS-CNRS \\ University of Toulouse, INP \\ Toulouse, France \\ Olivier.bernal@toulouse-inp.fr
}

\author{
Hélène Tap \\ $L A A S-C N R S$ \\ University of Toulouse, INP \\ Toulouse, France \\ helene.tap@toulouse-inp.fr
}

\begin{abstract}
We designed an integrated long period grating based on the horizontal periodic tapering of a silicon nitride channel waveguide. The structure is studied for gas sensing applications, using Styrene AcryloNitrileas sensitive layer. Simulation results from the proposed architectureshow ahigh sensitivity beyond1700 $\mathrm{nm} / \mathrm{RIU}$ using awavelength interrogation method centered at $1550 \mathrm{~nm}$ for a $20 \mu \mathrm{m}$ large and $300 \mu \mathrm{m}$ long structure.
\end{abstract}

Keywords-Integrated photonics, gas sensor, modeling, long period grating, coupled local mode theory.

\section{INTRODUCTION}

For the past two decades,long period fiber gratings (LPFGs) have beenintensely investigated for communication [1] and sensing applications [2]. A wide diversity of LPFGbased systems has been demonstrated and used in various applications, such as optical filters [3], gain flatteners, polarization couplers, multiplexers and particularly refractometric sensors [4-7]. However, to the best of our knowledge, the design of these structuresis typically limitedto standard dimensions and intrinsic parameters of the host fibers, rendering them unsuitable for integration and optimization.

Following the enormous impact of integrated electronics on our society, integrated photonicshave been subject to an exponentially growing interest since its earliest inception as laser diode devices for telecommunication applications in the 1960sand is now widely studied for the design of original, power-efficient and high-resolutioncompact sensors. In 2003, Tsoi and al. [8] demonstrated the first integration of a long period waveguide grating (LPWG)having similar properties to conventional LPFGs. The LPWGarchitectureusing a periodic perturbation of the width or height of the waveguide enables strong coupling between the fundamental mode and a copropagating cladding mode.It can thus be designed to selectively perform coupling under specific conditions, makingthem very interesting candidates for in-situ chemical monitoring. Using a suitablefunctionalizing chemical layer for the cladding window surrounding the LPWG core, the detection and quantification of a specific analyte has been demonstrated through various techniques[4,5,7].
The fabrication of index-modulated structures required for long period gratings can be performed through local corrugation [9], tapering [6], laser irradiation, ultraviolet inscription, arc discharge [10]. We propose here to introduce a grating perturbation on the fundamental mode of a simple channel waveguide through a smooth periodic change of the waveguide width.Unlike binary gratings, this type of grating enables smooth power coupling and can be studied underthe slowly-varying envelope approximation assumptions. The design is studied to analyzethe LPWG response to a change of analyte concentration through the change of the sensing materialrefractive index.

This paper is organized in three parts. First, in Section II, the general design of the horizontal LPWG is presented and compared to other reported types of LPWG. In Section III, the Coupled Local-Mode Theory (CLMT) [11] is used to introduce and describe the principle of the LPWG's mechanism This approach is formulated as a proof-ofconcept but can subsequently beextended to accurately model the LPWG response and characteristics[12,13]. In Section IV, CLMT is applied on our structure andan EigenMode Expansion (EME) method is performed with Lumerical suite tools as a comparison and simulation results of the sensor's sensitivity are discussed.Finally, in section $\mathrm{V}$,two fabrication methods are proposed.

\section{ARCHITECTURE}

\section{A. Proposed structure}

The proposed LPWG sensor structure consistsofa periodically-modulated(along the $\mathrm{z}$-axis) channel width( $\mathrm{x}$ axis) waveguide inside of a polymeric sensing regionas illustratedin Figure 1.

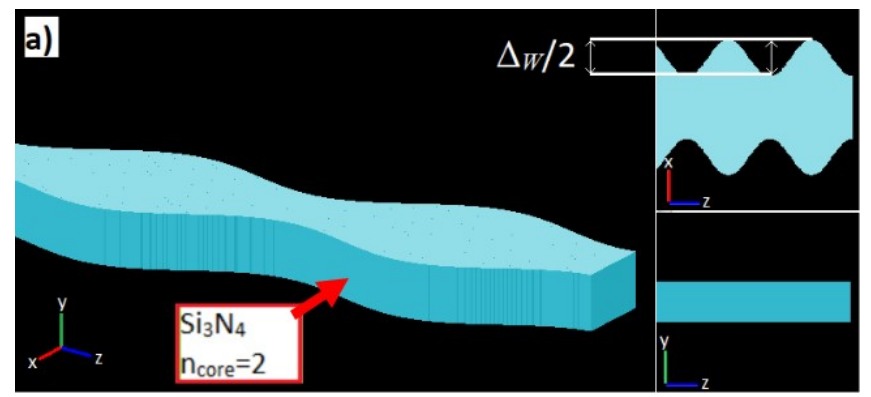




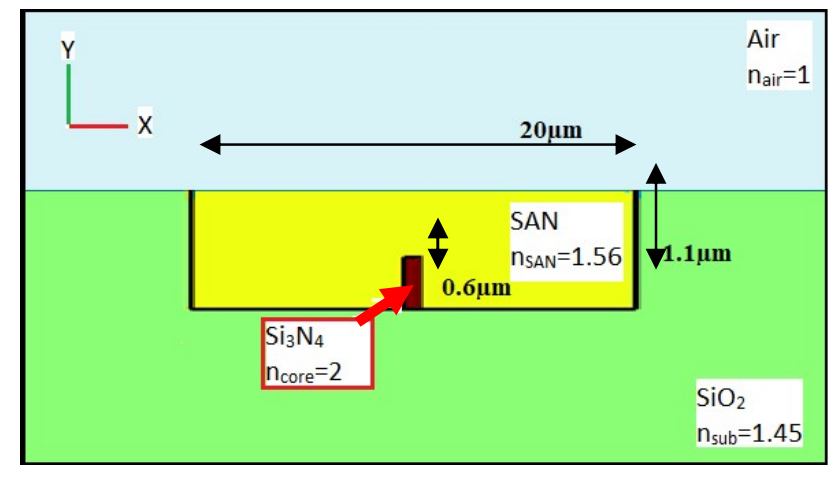

Fig. 1. (a)Horizontal LPWG coreprofiles and (b)cross-sectional material architecture where the Si3N4 and the Styrene AcryloNitrile(SAN) are the core and cladding waveguides respectively

As explained in the next section using CLMT [11], the excitationof the coupling phenomenon between core and cladding modes is performedvia a periodic modification of the local modes along the propagation $\mathrm{z}$-axis. Two different grating types can allow this mechanism. The most common grating is one with a local index variation inscribed in the waveguide or core $[1,10]$. It usually requires a specific and complex apparatus as well as accurate calibration on each material to be engraved. The second grating structure, more commonly employedin integrated photonics structures, are corrugatedwaveguides $[9,14]$, which can be achieved by an additional vertical etching step to createrelief, leading to optical coupling to cladding modes above the waveguide core. On the contrary, our structure is designed to incorporatea horizontal modulation via a periodic waveguide width variation and the design of a specific cladding box. Hence, no further process step is necessary. This is advantageous since the modulation pattern is directly drawn on the layout.

\section{B. Materials and dimensions}

The sensing mechanism is based on the coupling between two different modes; the fundamental core mode, HE1 of effective index $n_{\text {eff, } 1}$, and an $n^{\text {th }}$ cladding mode of effective index $\mathrm{n}_{\text {eff,n, }}$ which is mostly confined in the cladding whichcan later be functionalized to a specific chemical. Since grating-assisted coupling involves coupling the core mode to higher-order modes, which are modes with lower effective indices, the following conditionmust be fulfilledto have mode confinement

$$
\mathrm{n}_{\text {core }}>\mathrm{n}_{\text {eff }, 1}>\mathrm{n}_{\text {clad }}>\mathrm{n}_{\text {eff, }}>\mathrm{n}_{\text {sub }}>\mathrm{n}_{\text {air }}
$$

where $\mathrm{n}_{\text {core }}, \mathrm{n}_{\text {clad }}, \mathrm{n}_{\text {sub }}$ and $\mathrm{n}_{\text {air }}$ are the refractive indexes of the $\mathrm{Si}_{3} \mathrm{~N}_{4}$ waveguide core, the cladding, the $\mathrm{SiO}_{2}$ substrate, and the air respectively.

Aspecific set of materials and structure dimensions must thenbeconsidered to allow both standard manufacturing process and functionalization for potential gas sensing. Silicon nitride $\left(\mathrm{Si}_{3} \mathrm{~N}_{4}\right.$ or more commonly $\left.\mathrm{SiN}\right)$ isemployed for its ease of growth in the domain of integrated photonics and optical properties(i.e. for its relatively mediumindexcontrast with silicon oxide). The sensitive layeremployed as cladding must be a porous polymer, in which the gas is soluble.In addition, the sensitivity and selectivity can be enhanced by employing supramolecular compounds which have a cage-like structure within which molecules can be reversibly trapped [21].For example, Cryptophane-A which presents the smallest relative cavity, a methane trap,can be incorporated into a Styrene
AcryloNitrile (SAN) film of index $n=1.56$, forming the sensing layer of a Mach-Zehnder-based methane sensor[15,16]. It is referred to here as cladding, as it almost entirely encompasses the Silicon Nitride core(Fig. 1.(b) and 2.(a))

Figure 1. (b) describes the cross-sectional refractive index profile of the LPWG, with $\mathrm{n}_{\text {core }}=2, \mathrm{n}_{\text {clad }}=1.56$, $\mathrm{n}_{\text {sub }}=1.45, \mathrm{n}_{\text {air }}=1$. A sensing window thickness of $\sim 1$ $\mu$ mallows uniform and relatively quick diffusion of the gas species to be detected as demonstrated in $[5,7,8,15]$. The cladding and waveguide are respectively designed to have $\mathrm{a} 20$ and 0.8 ( $\left.w_{\text {core }}\right) \mu \mathrm{m}$ width and a $1.1 \mu \mathrm{m}$ and $0.6\left(h_{\text {core }}\right) \mu$ mthicknessto ensure single mode propagation only within the core while allowing multiple higher order modes within the cladding.Moreover, the cladding dimensions determine the maximum order of the propagating cladding modes, which directly influences the coupling strength, as demonstrated later in section III and IV.Themaximum width variation $\Delta_{W}$ (see Fig. 1. (a))and grating length period $\Omega$ are chosen to be $0.6 \mu \mathrm{m}$ and $8.76 \mu \mathrm{m}$, respectively (Fig. 1 a)so that $\Omega$ corresponds to the standard LPG phase-matching condition $[11,12,13]$ on thepropagationconstants $\beta_{j}(z)$ and $\beta_{l}(z)$ of the two modes to couple at the resonance wavelength around $1550 \mathrm{~nm}$ :

$$
\beta_{j}(z=0)-\beta_{l}(z=0)=\frac{1}{\Omega},
$$

Here, coupling occurs between the fundamental mode HE1, andhigher order propagating cladding modes, out of which one exhibits a higher coupling at a specific wavelength as described in the next section.More precisely, it will be further shown that only odd cladding modes can be coupled to the fundamental mode through the LPWG.Their respective effective indices and profiles were simulated with Lumerical,as shown in Fig. 2 for 2 higher-order modes only, to calculate the resonant grating period. We see that for the two multi-modes, most of the optical field is confined into the SAN, therefore called cladding-modes. These modes thus become inherently more affected by a change of the

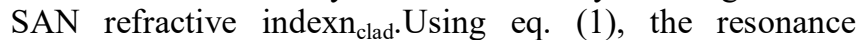
wavelength can be approximated by the average of the values of each coupled-mode effective indicesalong $\mathrm{z}$ propagation. Note, however, that the real resonance wavelength values will be slightly shifted as the functions of the mode effective indicesversuscore width are not linear.

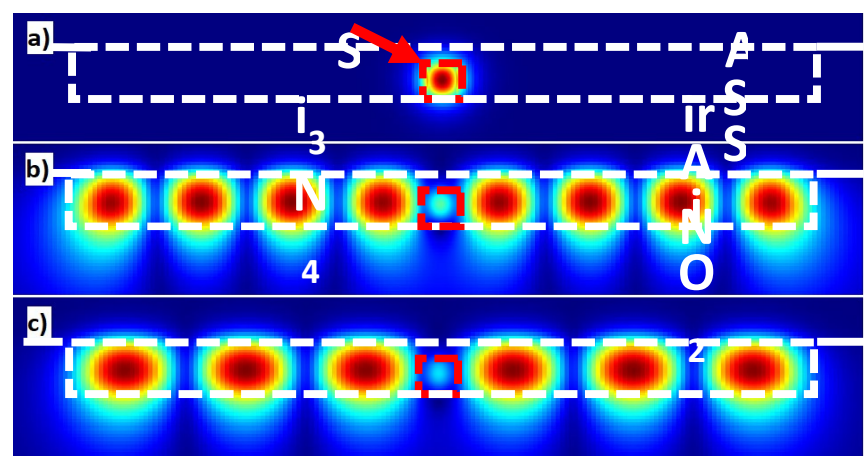

Fig. 2. Mode profiles at 1550nm for Fundamental (a)HE1 mode, and cladding (b)HE9 and(c) HE7 modes calculated with Lumerical mode solver 


\section{LPG MODEL}

Different methods have been used to model the light propagation when introduced into the LPG. However, certain types of simulation methods find difficulty to model accurately this type of sensor as the behavior of the distribution of light is very continuous and highly depends on small structure variations.

\section{A. Finite-Difference Time-Domain}

As an example, Finite-Difference Time-Domain (FDTD) gives an interesting insight of the overall sensor mechanism as shown in Fig 3, where coupling happens between HE1 and HE7, but its high dependence onfine discretization makes it inadequate for accurate modeling in our case using our computing resources. The need for both high temporal and mesh resolution over a relatively large area, especially at the interfaces where refraction happens, leads to large memory needs, otherwise simulations will present larger numerical errors than other methods. However, comparing Fig. 3. (a) and (b) clearly illustrates that, only at resonance, most of the optical power is transferred from the core mode to the higher order cladding mode after a brief propagating length.
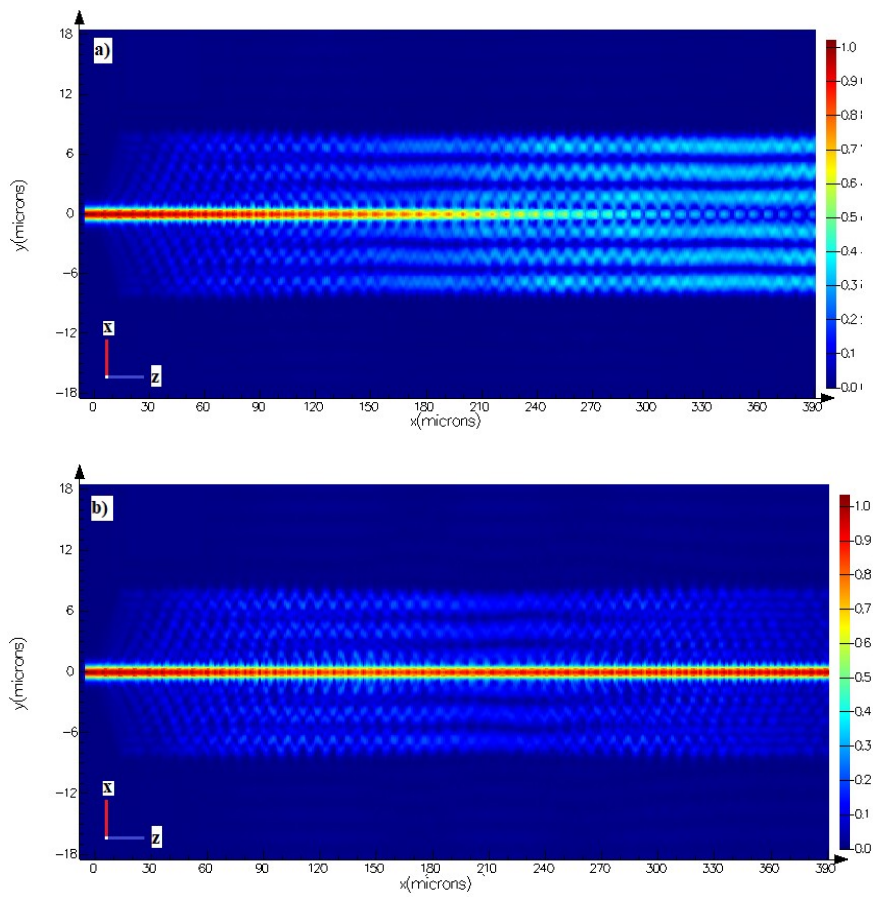

Fig. 3. (a)Illustration of the intensity distribution seen from above at resonance (b)Normalizedintensity distribution seen from above with a slightly different cladding index

To circumvent these computing issues, two different methods can be used instead: the common EigenMode Expansion method and, more specific to our LPG application, the CLMT [11].

\section{B. Eigenmode Expansion method}

The Eigenmode expansion method (EME), also referred to as mode matching method, is a rigorous technique used for modeling waveguides $[17,18]$, which relies on a scattering parameter superposition approach to calculate the amplitude of each mode at any propagation distance. This technique has very good efficiency for periodic and/or long components and provides flexible post-calculations analysis. It is thus ideal for modeling long period grating-based systems [18] as, due to the structure periodicity, it is possible to calculate the structure scattering parameters fromone single period. The method is available in various commercially available software such as Lumerical(C) suite tools, which was used to simulate the results presented on fig. 2 .

Figure 2. shows (a) the fundamental mode profile and (b)-(c) the profile of two cladding modes that can couple to the fundamental mode at certain conditions. The gratingperiod is longitudinally divided into 40 block objects and the guided mode calculation considers 40 modes per block similarly to [12].

\section{Coupled Local-Mode Theory application}

On the other hand, the CLMT application is performed through the characterization and calculation of the continuous refraction at the interfaces between the core and sensing window, which providesa more physical model on the coupling behavior in the LPG. The application of the theory was performed throughMatlab $\subset$ and COMSOLC linked scripts.

Conventional Coupled Mode Theory (CMT)[11] is usually used to describe the coupling between modes when small perturbations are assumedand modes remain almost unchanged along the propagation direction.Further, the general coupled mode equations are restricted to weakly guiding waveguides where polarization effects are ignored.CMT can thus model very accurately the first category of LPGs mentionedin Section II, where the waveguide dimensions remain constant and only local refractive indices are modified. However, in the presenceof significant cross-sectional dimension modulations [13], CMT can no longer be applied, and a more specific approach, such as CLMT [11], is required. CLMT achieves good results $[12,13]$ when used to describestructures with large but slowly varying non-uniformitiesalong the waveguide and can thus be potentially used to describe our structure. In this section, a brief introduction to CLMT is formulated together with a method to calculate coupling coefficients. It will allow better understanding of the structure behavior and provide more insightto further optimize the design architecture.

Generally, there isno exact solution to Maxwell's equations for propagation along a perturbed waveguide.It is, however, possible to obtain accurate approximations of modes within local regions, known as local modes [11]whichsuperpose to form the transverse field components as

$$
\Phi_{t}(x, y, z)=\sum_{j}\left\{b_{j}(z)+b_{-j}(z)\right\} \hat{\varphi}_{t j}\left(x, y, \beta_{j}(z)\right),
$$

where $\hat{\varphi}_{t j}$ representsthe orthonormal electric or magnetictransverse field profiles of the $j^{\text {th }}$ mode, and $b_{ \pm j}$ (z)areboth modal amplitude and phase for forward $(+)$ or backward (-) propagation.

The coupling behavior between local modes is formulated by the general coupled local-mode equation [11] 


$$
\left.\frac{d b_{ \pm j}}{d z} \mp i \beta_{j} b_{ \pm j}=\sum_{j}\left\{C_{ \pm j, l} b_{l}+C_{ \pm j,-l} b--l\right)\right\},
$$

with $C_{j l}$ the coupling coefficient between the local modes $j$ and $l$ which can be expressed as[11]

$$
C_{j l}=\frac{1}{4}\left(\frac{\varepsilon_{0}}{\mu_{0}}\right)^{\frac{1}{2}} \frac{1}{\beta_{j}-\beta_{l}} \int_{A \infty} \hat{e}_{j}^{*} \cdot \hat{e}_{l} \frac{\partial n^{2}}{\partial z} d A, \quad j \neq l ; \quad C_{j j}=0
$$

The squared index component of the horizontal grating structure can be expressed here as

$$
n^{2}(x, y, z)=\left\{\begin{array}{ll}
n_{\text {clad }}^{2} & y>\frac{h_{\text {core }}}{2} \\
n_{\text {core }}^{2}\left(1-\Delta_{n} H(x-\rho(z))\right. & |y| \leq \frac{h_{\text {core }}}{2} \\
n_{\text {box }}^{2} & y<-\frac{h_{\text {core }}}{2}
\end{array}\right\},
$$

with $\Delta_{n}=1-\frac{n_{\text {clad }}^{2}}{n_{\text {core }}^{2}}, \rho(z)=\frac{\Delta_{w}}{4} \sin \left(\frac{2 \pi z}{\Omega}\right)+\frac{w_{\text {core }}}{2}$ and where $x=y=0$ at the center of the waveguide. $H$ denotes the Heaviside function and $\rho$ the periodic width variation of the LPWG along the $\mathrm{z}$ axis. By substituting eq. (5) into (4), we obtain the coupling coefficient for localmodes of an integrated horizontalgrating in the form of

$$
C_{j l}(z)=\frac{1}{4}\left(\frac{\varepsilon_{0}}{\mu_{0}}\right)^{\frac{1}{2}} \frac{n_{\text {core }}^{2} \Delta_{n}}{\beta_{j}-\beta_{l}} \frac{d \rho(z)}{d z}\left[\begin{array}{l}
\frac{h_{\text {core }}}{2} \\
\left.\int_{\frac{h_{\text {core }}}{2}}^{2}\left(\hat{e}_{j}^{*} \cdot \hat{e}_{l}\right)_{x=\rho(z)} d y\right) \\
\frac{h_{\text {core }}}{2} \\
+\int_{-h_{\text {core }}}^{2}\left(\left(\hat{e}_{j}^{*} \cdot \hat{e}_{l}\right)_{x=-\rho(z)} d y\right)
\end{array}\right],
$$

with $C_{j j}=0$.

Further, the transverse electric field at the interfaces of a step-index waveguide of relatively high index contrast is discontinuous, which complicates the calculation of the integrand. A bypass method [11] is to recognize that $n^{2} e_{t}$ is continuous across the interfaces. As a result, the transverse component of the integrand can be replaced by

$$
\left(\frac{n^{2} \hat{e}_{t, j}^{*}}{n_{\text {core }}^{2}}\right) \cdot\left(\frac{n^{2} \hat{e}_{t l}}{n_{\text {clad }}^{2}}\right)
$$

As previously mentioned, one of the main advantages of this CLMT compared to the EME method consists in providing a better insight of the phenomena taking place in the LPG. In particular, important LPWG properties can be deduced from this equation (eq. 6):(1) onlycoupling of the fundamental mode to odd cladding modes is possible as the even mode coupling coefficients are null;(2) the coupling amplitude can be increasedby modifying the grating amplitude $\Delta_{W}$ and period $\Omega$ (via $\rho$ ) or by optimizing the field superposition at the waveguide interfaces. The increase of the coupling coefficient amplitude fastens the rate at which energy is exchanged between modes, as described in eq. (3), thereby reducing the minimumrequiredLPWG's length to achieve maximum coupling.
To obtain a good estimation of the remaining integral in $C_{j l}(z)$ (eq. 6), COMSOL mode-solver, based on finiteelement method, can be used as it allows a convenient way to extractthe interface fields of each modes, their effective indices, and Poynting vectors for normalization. Ten crosssections were simulated by sweeping the waveguide width between minimum and maximum values to constitute a dataset of values. These calculations are only required over half a period of the 3D model because of the structure's periodicity. This dataset is then transferred to Matlab, coupling coefficients are calculated and extracted, and only then, the LPG geometry is taken into account to model energy distribution. Another advantage of the proposed model based on CLMT is thatusing this predetermined dataset, any LPG geometry can be designed and simulated easily. CLMT only requires a dataset of coupling coefficients for given cross-sections and directly calculates energy distribution as a function of $C_{j l}(z)$, while EME superposes each mode to calculate scattering parameter between each section.

\section{SIMULATIONRESULTS}

\section{A. Model validation}

Figure4 shows the cladding mode power versus the propagating length along the LPWG for different wavelengths obtained withbothCLMT and EME techniques. It clearly shows that comparable results can be achieved with the CLMT.In addition, here, the highest order propagating mode (HE9, for our LPWG's dimensions)shows higher coupling than anylower order modes (e.g. HE7) at their respective resonant wavelengths (HE9@1540nm and HE7@1484nm), as previously demonstrated by the CLMT equations, due to a higher field product integrand at the core/cladding interface. This results in a reduced LPWG's length to achieve maximum coupling.The "HE9 normalized power" at wavelengths $1550 \mathrm{~nm}$ and $1525 \mathrm{~nm}$ clearly describes the coupling behavior of the LPWG when the injected wavelength drifts from the resonance, resulting in a reduction of the coupled energy at a the optimal sensor length (the shortest length where coupling reaches its peak at resonance), $\mathrm{L}_{\text {opt }}=300 \mu \mathrm{m}$ here.

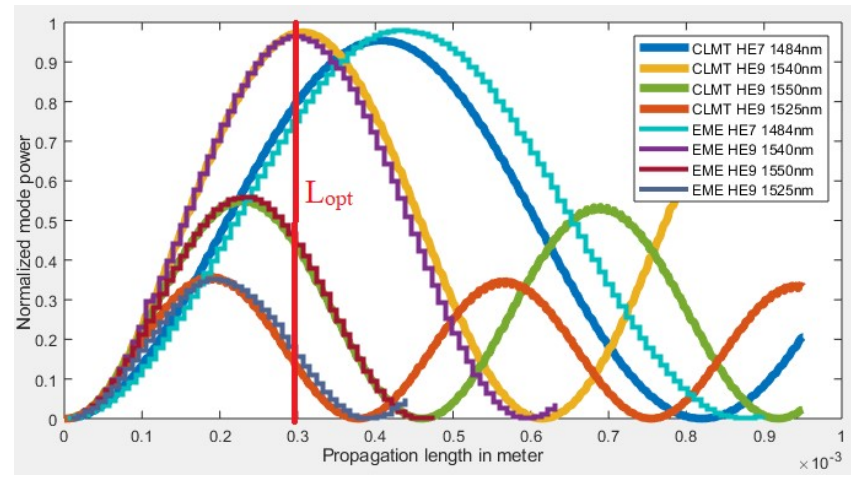

Fig. 4. Cladding mode normalized power versus propagation length (SI) forHE7 and HE9 modes at different wavelengths simulated by CLMT application and EME

\section{B. Sensor sensitivity}

The system interrogation can be performed through the wavelength interrogation method [4-7]. The refractive index 
sensing mechanism relies on the disruption of the resonance of the LPWG which occurs when the cladding mode propagation constant changes. As a main difference with typical LPFG chemical sensors, which usuallyinvolve SPR as the cladding material cannot be modified easily, the cladding mode here is mostly confined in the sensing layer incorporated with the selective transducer. It is expected to lead to a relatively large modification of the mode propagation constant when the material indexchanges, i.e.analyte-inducedperturbations, which enhances sensor sensitivity.

Figure 5. (a) shows the coupling spectrafordifferent cladding refractive indices. From these results, thesensitivity can be calculated (cf. Fig. 5. (b)). The average sensitivity is found to be $1768 \mathrm{~nm} / \mathrm{RIU}$ (refractive index unit).
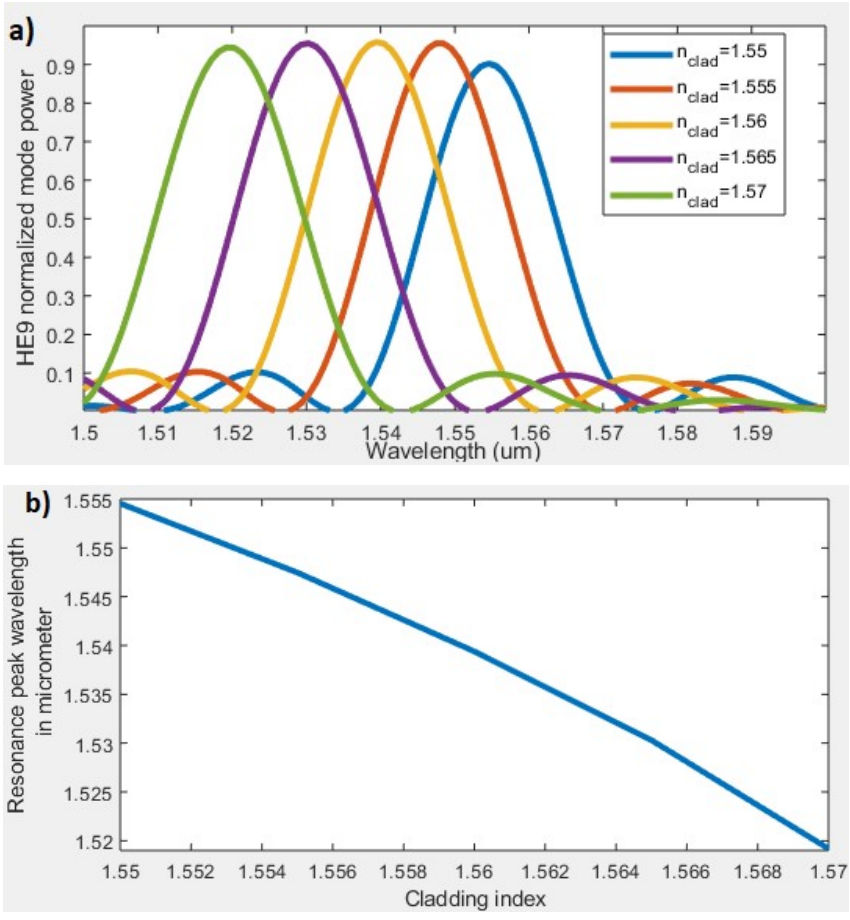

Fig.5. (a) HE9 normalized mode power spectra for a 33 periods long grating at different cladding refractive indices, calculated with EME method and (b) resulting resonance peak shift for wavelength interrogation

As it is difficult to measure the output spectra of the higher mode the measurement is made directly at the output of the core, which allows to get the same wavelength via detecting a dip in the light in the core.

We assume here that the output core intensity is equal to the input intensity minus the coupled energy to cladding modes along propagation, which could easily be measured using grating couplers, for example.

One can also notice a LPG peak is larger than other resonance peak found in integrated optics, which prevents it from large linewidth source issues.

\section{FABRICATION PROPOSALS}

This new type of integrated refractometric sensor is based on a specific coupling mechanism which allows several profile configurations, and therefore multiple realization processes. As described in the first section, the effective indices of each region must respect a certain relationship. Two different approaches can be employed to fabricate the LPWG.

A first method would be to simply create an opening into the buried core of a photonic chip and cover it with a sensing film to fill the etched region as seen in Fig. 6. (a). A downsize to this method is the lack of control of the thickness of the film at certain areas where the cladding could form a rib multi mode waveguide instead of a strip one. It could simply be bypassed to a certain extent by performing spin coating to different speeds until the thickness reaches a value corresponding to a desired $\mathrm{n}_{\mathrm{eff}, \mathrm{n}}$ of the rib cladding mode.

A second method (Fig. 6. (b)) currently being investigated would be to perform lithography on the polymer layer, creating polymer waveguides encompassing the core waveguide. (19) This type of structure could also be translated to an electro optical multi-mode coupler, enabling selected coupling of the fundamental mode into a specific higher order mode.

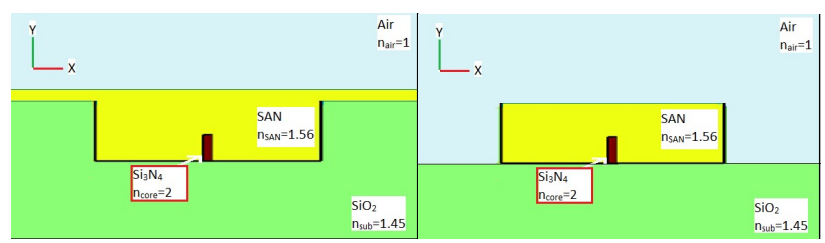

Fig. 6. (a)Rib cladding structure and (b)Strip cladding structure

\section{CONCLUSION}

We have presented a new sensor architecture based on a long period horizontal gratingstructure. We have also proposed to describe the LPWG using the CLMT which provides more insight on the physics of the structure.For example,it indicates that a higher index contrast and therefore higher $n_{\text {core }}$ values could increase the coupling, rendering the architecture an interesting candidate for silicon on-chip integration. This approach has been successfully compared to and verified using a standard method based on EME. Moreover,CLMT is more flexible to common grating profile modulations $[12,20]$ as we work on the reconstruction of the coupling coefficient shape along propagation. The calculated overall sensor sensitivity reaches $1768 \mathrm{~nm} / \mathrm{RIU}$, and further improvement could increase the sensitivity to over $2000 \mathrm{~nm} /$ RIU. Based on [21], from the achieved Mach Zehnder sensitivity of 0.768 $\mathrm{mrad} / \mathrm{ppm}$, the SAN and Cryptophane layer sensitivity to methane in terms of RIU/ppm canbe estimated to be approximately $6.3 \mathrm{nRIU} / \mathrm{ppm}$. As a result, using a similar sensitive layer with our proposed LPG architecture, an 11 $\mathrm{fm} / \mathrm{ppm}$ theoretical wavelength sensitivity could be expected.This new type of design appears promising and can benefit from improvements or hybridations, potentiallyinvolving new sensing mechanisms, like plasmonics[18] or photonic crystals[22]. 
[1] K.S .Chiang and Q. Liu, "Long-period grating devices for application in optical communication," 2006.

[2] H. Tsuda and K. Urabe, "Characterization of long-period grating refractive index sensors and their applications, Sensors vo.9, 2009

[3] A .M. Vengsarkar, P .J. Lemaire, J.B. Judkins, V. Bhatia, T. Erdogan and J.E. Sipe, "Long-period Fiber gratings as band-rejection filters", Journal of Lightwave Technology, vol. 14, no. 1, pp. 58-65, June, 2007.

[4] J.A. Barnes, R.S. Brown, A.H. Cheung, M.A. Dreher, G. Mackey and H.-P. Loock, "Chemical sensing using a polymer coated long-period fiber grating interrogated by ring-down spectroscopy", Sensors and Actuator B 148, April 2010.

[5] J.A. Barnes, M.A. Dreher, K. Plett, R.S. Brown, C.M Crudden and H.-S. Loock "Chemical sensor based on a long period fibre grating modified by a functionalized polydimethylsiloxane coating", The Analyst,vo. 133, no. 11 pp. 1541-1549, November, 2008

[6] W.B. Ji, S.C. Tjin and C.L Ng,'Highly sensitive refractive index sensor based on adiabatically tapered microfiber long period gratings", Sensors vo.13, 2013

[7] S. W. James, S. Korposh, S.-W. Lee and R.P.Tatam, "A long period grating-based chemical sensor insensitive to the influence of interfering parameters" Optics express vol. 22, no.7, April, 2014.

[8] H.C. Tsoi, W. H. Wong and E.Y.B. Pun, "Polymeric long-period waveguide gratings", IEEE Photonics technology letters, vol. 15, no. 5, May, 2003.

[9] S. Pal, A. Chauhan, M. Singh, P. Kumar, M. Sharma, N. Pradhan, K. Singh and C. Dhanavantri, "Realization of long-period corrugated grating in silica-on-silicon based channel Waveguide", IEEE photonics Technology Letters, vol. 21, no. 20, October 2009.

[10] J.M. Estrudillo-Ayala, R.I. Mata-Chavez, J. C. Hernandez-Garcia, R. Rojas-Laguna, "Long period fiber grating produced by arc discharges", Fiber optic sensors, 2006, pp. 295-313.

[11] Allan W. Snyder and John D. Love, Optical waveguide theory. London, New York: Chapman and Hall, 1983

[12] Qingning Li,"Coupled-local-mode theory and study of optical peoperties of a gaussian fiber grating", J. Opt. Soc. Am.A, vol. 16, vo. 6, June 1999.

[13] Long Jin, Wei Jin, Jian Ju, and Yiping Wang, "Coupled local-mode theory for strongly modulated long period gratings", Journal of lightwave technology, vol. 28, no. 12, June, 2010.

[14] D. Zhang, Y. Zhang, Y. Cui, C. Chen, and E.Y.B. Pun, "Long period grating in/on planar and channel waveguides: a theory description", Optics \& Laser Technology 39 1204-1213, September, 2006.

[15] F. Dullo, S. Lindecrantz and J. Jagerska, "Sensitive on-chip methane detection with a cryptophane-A cladded Mach-Zehnder interferometer" ,Optics express vol. 23, no.24, November, 2015.

[16] Haris Apriyanto. "Study, analysis and experimental validation of fiber refractometers based on single-mode, multimode and photonic crystal fibers for refractive index measurements with application for the detection of methane.", Hal archives-ouvertes March, 2019.

[17] EME. [online] Kb.lumerical.com. Available at: https://kb.lumerical.com/solvers_eigenmode_expansion.html

[18] Yue Jing He, "Investigation of LPG-SPR sensors using the finite element method and eigenmode expansion method", Optics express vol. 21 no. 12, June 2013.

[19] Martin F. Broglia, Diego F. Acevedo, Denise Langheinrich, Heidi R. Perez-Hernandez, Cesar A. Barbero, and Andrés F. Lasagni, "Rapid Fabrication of Periodic Patterns on Poly(styrene-co-acrylonitrile) Surfaces Using Direct Laser Interference Patterning," International Journal of Polymer Science, vol. 2015, Article ID 721035, 7 pages, 2015.

[20] T. Erdogan, "Cladding-mode resonances in short- and long-period fibers," J. Opt. Soc. Am. A, vol. 14, no. 8, August 1997.

[21] Martin Ingvaldsen "Sensitivity to pressure and methane of a cryptophane-A doped polymer" semanticscholars, 2015

[22] P. Steinvurzel, E.D. Moore, E.C. Magi, B.T. Kuhlmey, and B.J. Eggleton, "Long period grating resonances in photonic bandgap fiber", Optics express vol. 14, no.7, April, 2006. 